\title{
From EIT photon correlations to Raman anti-correlations in coherently prepared Rb vapor.
}

\author{
Vladimir A. Sautenkov ${ }^{1,2}$, Yuri V. Rostovtsev ${ }^{1}$, and Marlan O. Scully ${ }^{1,3}$ \\ 1 Institute for Quantum Studies and Department of Physics, \\ Texas A \& $M$ University, 77843 \\ ${ }^{2}$ Lebedev Institute of Physics, Moscow 119991, Russia \\ 3 Princeton Institute for the Science and Technology of Materials and Department of Mechanical E Aerospace Engineering, \\ Princeton University, 08544
}

(Dated: November 9, 2018)

\begin{abstract}
We have experimentally observed switching between photon-photon correlations (bunching) and anti-correlations (anti-bunching) between two orthogonally polarized laser beams in an EIT configuration in $\mathrm{Rb}$ vapor. The bunching and anti-bunching sswitching occurs at a specific magnetic field strength.
\end{abstract}

PACS numbers: $32.80 . \mathrm{Qk}, 42.65 . \mathrm{Dr}, 42.50 . \mathrm{Hz}$

Interaction of macroscopic laser fields with a resonant atomic 3-level Lambda system has been the focus of recent research. In particular, interesting phenomena such as photon-photon correlation, phase squeezing, and new cenitangled states of the radiation field have been studled 1, 2, 3, 4, 5]. Sub-Poissonion statistics and a reduction of noise below photonic short noise have been demonstrated [4]. 'New bright source of entangled photon states with controable coherent time has been demonstrated [3] by employing parametric generation with counter-propagating electromagnetic waves in $\mathrm{Rb}$ vapor. The photon statistics of the light cemitted from atomic ensemble into a single field mode of an Ioptical cavity has been studied in [6], smooth transition from bunching to anti-bunching has been experimentally demonstrated by changing number of atoms in the cavity.

In this Letter, we study intensity correlations and anticorrelations of optical fields propagating through a dense Rubidium vapor. The main result of the paper is shown in' Fig. 1. For lower level coherence between Zeeman sublevels prepared by laser beams with orthogonal polarizations Figig. 1a), we observe that the intensity fluctuations of two laser beams are correlated under the condition of electromagnetically induced transparency (EIT) (see in Fig. 1b) and anti-correlated when two-photon detuning is introduced (see in Fig. 1c). That is, we have observed an interesting transition between bunching and anti-bunching by changing two-photon detuning. The two-photon spectral width of this transition is narrower than the width of the EIT window. A theoretical approach similar to that developed in [7, 8] has been used to explain the observed results.

An experimental setup is shown in Fig. 1a. All measurements have been performed with an external-cavity diode laser (ECDL) tuned to the center of the Doppler broadened $D_{1}$ line $\left(5 S_{1 / 2}(F=2) \leftrightarrow 5 P_{1 / 2}\left(F^{\prime}=1\right)\right)$. The output laser beam is split into two by a beam-splitter, then the polarization of the one of the beams is rotated by a $\lambda / 2$-wave plate, and these two orthogonally linear polarized beams are combined together by a polarizing beam-splitter (PBS). After the $\lambda / 4$ wave-plate the beam is a combination of two (a)

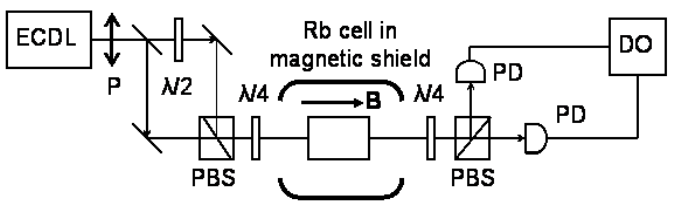

(b)

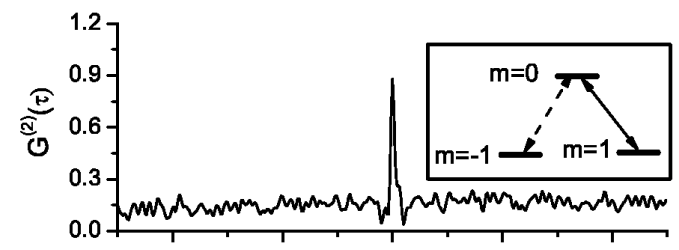

(c)

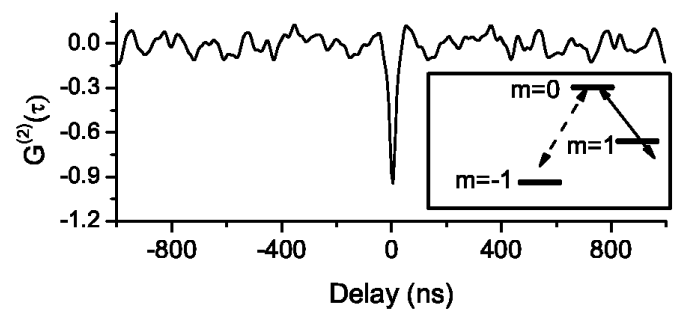

FIG. 1: (a) Experimental setup. ECDL is the extended cavity diode laser, $\mathrm{P}$ is the polarizer, $\mathrm{PBS}$ is the polarizing beam splitter, $\mathrm{PD}$ is the photo diode, DO is the digital oscillascope. Intensity correlation functions, $G^{(2)}(\tau)$, are shown corresponding to two cases: (b) no magnetic field, EIT, and (c) with magnetic field, $B=-0.47$ Gauss. It is shown in insets the simplified energy levels without and with magnetic field.

orthogonal circularly polarized optical fields which induce a ground state Zeeman coherence in $\mathrm{Rb}$ atoms. A simplified $\Lambda$-scheme is depicted in insets of Fig. 1(b,c). A glass cell $(l=7.5 \mathrm{~cm})$ with $\mathrm{Rb}$ vapor at density $10^{12} \mathrm{~cm}^{-3}$ has been installed in a two-layer magnetic shield.

After the cell, the transmitted laser beams with opposite circular polarizations are separated by a second $\lambda / 4$ 

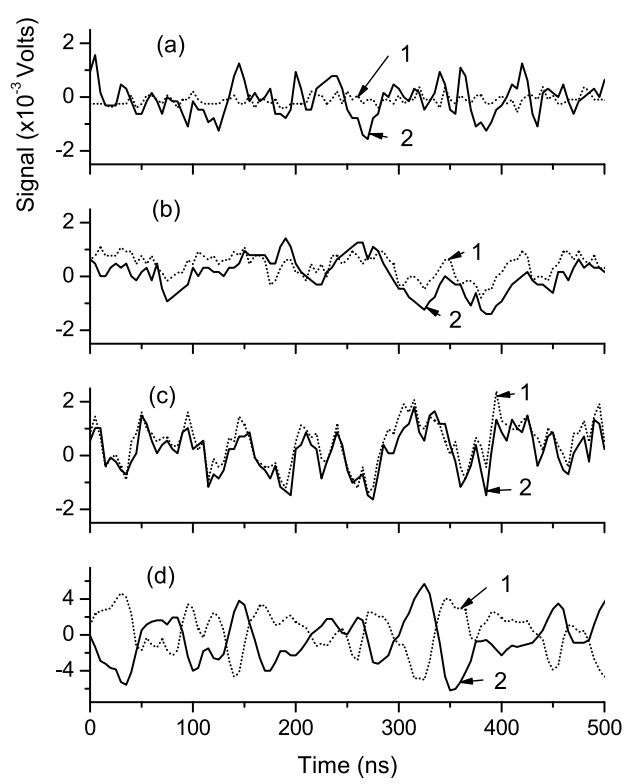

FIG. 2: Waveforms from photo-detectors with optical power of laser beams at front window of $\mathrm{Rb}$ cell is $0.5 \mathrm{~mW}$. The fluctuaions of intensity of (a) one beam (the second is blocked) before and after the cell, (b) two spatially separated beams, (c) two coinciding beams without magnetic field $(B=0)$ at the EIT, zero two-photon detuning and (d) for magnetic field $B=-0.47$ Gauss.

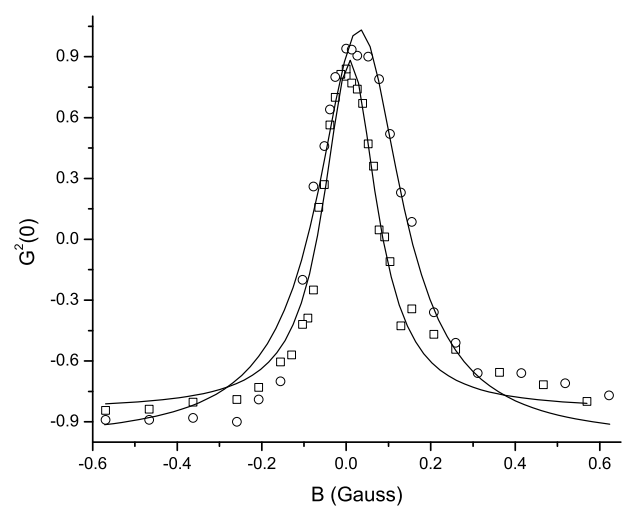

FIG. 3: Correlation $G^{(2)}(0)$ versus magntic field (two-photon detuning). Circles corresponds to optical power $0.5 \mathrm{~mW}$, squares - optical power $0.25 \mathrm{~mW}$.

wave-plate and another polarizing beam splitter. Transmitted laser beams were focused on fast photodiodes (PD) ET 2030A (Electro-Optics Technology) with frequency bandwidth $75 \mathrm{kHz}-1.2 \mathrm{GHz}$. The optical path lengths for both beams are the same. Signals from PDs are analyzed by a digital oscilloscope (DO) (Agilent 54624A with frequency bandwidth $100 \mathrm{MHz}$ ).

At the optical power of each beam at the entrance window of the $\mathrm{Rb}$ cell is $0.5 \mathrm{~mW}$, beam diameters $0.1 \mathrm{~cm}$, we vary a magnetude of longitudinal magnetic field and observe change in transmission. Linear transmission is less than $1 \%$, and at maximum of EIT transmission is 0.63. The measured FWHM is 0.86 Gauss that is five times broader than for a low power limit 0.16 Gauss, i.e. the width of EIT is determined by strong power broadening. By using ground state Zeeman splitting $\Delta \omega / 2 \pi_{B}=0.7 \mathrm{MHz} /$ Gauss, one can estimate the spectral width of the EIT resonance of $1.2 \mathrm{MHz}$ that are several times narrower than natural FWHM of hyperfine transitions $6 \mathrm{MHz}$ and Rabi frequency.

To study fluctuations of optical fields transmitted through the dense $\mathrm{Rb}$ vapor, we have registered the dependence of the intensities of both optical beams on time, $\left\langle I_{1,2}\right\rangle+\delta I_{1,2}(t)$. Here $\left\langle I_{1,2}\right\rangle$ are the average intensities of the laser beams, and $\delta I_{1,2}(t)$ are the time dependent intensity fluctuations shown in Fig.2. Data presented in Fig. 2 are a part of the 10 $\mu$ sec recorded data. The signal in Volts is proportional to laser intensity as $500 \mathrm{~V} / \mathrm{W}$. Waveforms shown in Fig.2a are obtained under the condition that when one of the beams is blocked before the cell and there is no magnetic field $(B=0)$. The first curve shows intensity fluctuations of optical beam before it goes into the cell, it has practically no amplitude noise, and in order to see optical intensity noise above the photodetector noise the optical power has to be increased at least three times. The second curve (with a larger waveform amplitude) shows excess noise of transmitted optical fields induced by the dense $R b$ vapor.

Waveforms in Fig.2b show intensity fluctuations of two separated optical beams. Beams are separated by a tilted glass plate (an incident angle is close to the Brewster angle) with good quality parallel optical surfaces. Distance between beams is $0.3 \mathrm{~cm}$ that is bigger than the beam diameters. One can see that the vapor induced noise is slightly correlated. Correlation increases when separation is reduced. Strong correlation between signals is shown in Fig.2c under resonance EIT conditions. In Figure $2 \mathrm{~d}$ waveforms are recorded with two-photon detuning by applying a magnetic field $B=-0.47$ Gauss.

One can see from Fig.2 that there are strong correlations and anticorrelations between beams governed by the EIT condition. The correlation function $G^{(2)}(\tau)$ between intensities of two optical beams is calculated by

$$
G^{(2)}(\tau)=\frac{\left\langle\delta I_{1}(t) \delta I_{2}(t+\tau)\right\rangle}{\sqrt{\left\langle\left[\delta I_{1}(t)\right]^{2}\right\rangle\left\langle\left[\delta I_{2}(t+\tau)\right]^{2}\right\rangle}}
$$

where averaging over the time is defined as $\langle Q(t)\rangle=$ $\int_{t}^{t+T} Q(t) d t / T, \tau$ is the selected time delay between the recorded signals, $T$ is the time of integration, in our case $T=10 \mu \mathrm{s}$.

In Figure 1b, the curve is calculated by using waveforms recorded under resonance EIT condition $(B=0)$. The correlation peak at delay time $\tau=0$ has an amplitude of 0.9 and the background average value near 0.15. Figure 1c demonstrates a pronounced modification of waveforms due to twophoton detuning (applied magnetic field $B=-0.47$ Gauss) showing that the waveforms are anti-correlated. For the reduced optical power, the width of the correlation peaks has 
decreased. The widths of the peaks is associated with the saturated width of resonance in $\mathrm{Rb}$ vapor absorption (a single photon resonance) [9].

To study the role of coherence and to ensure that the results obtained are related to the interaction between optical beams we perform experiments with separated beams. The results of analysis for power of $0.5 \mathrm{~mW}$ show that the correlation $G^{(2)}(\tau)$ obtained with spatially separated beams has the background 0.3 and a correlation peak with magnitude 0.7 at the delay $\tau=0$. When we reduce the spatial separation between beams, the contrast and the magnitude of the correlation pick is increased.

We perfom a set of measurements of $G^{(2)}(0)$ for different values of the magnetic field $B$. The correspondent dependence vs magnetic field for optical powers $0.5 \mathrm{~mW}$ and 0.25 $\mathrm{mW}$ are shown in Fig.3. At zero magnetic field $(B=0)$ the correlation between $\delta I_{1}(t)$ and $\delta I_{2}(t)$ is close to 0.9 . The correlation decreases with increasing of the xmagnetic field, it goes through zero, and then it reaches magnitude -0.9 .

Intensity correlation $G^{(2)}(0)$ demonstrates a resonance-like behavior on the change of magnetic field. The widths of these resonances are 0.24 and 0.16 Gauss correspondingly that is nearly 4 times narrower than the width of the EIT window at the same optical power. The EIT width (FWHM) increases from 0.65 Gauss to 0.86 Gauss with optical power of the each laser beam from 0.25 to $0.5 \mathrm{~mW}$. The smallest measured width of the correlation resonance coincides with the width of the EIT resonance at the low power limit 0.16 Gauss.

The intensity fluctuations can be described in various ways, e.g. by the Heisenberg-Langevin approach, a FokkerPlanck analysis, the quantum regression theorem, etc. [18], each provides their own insights to the classical and quantum fluctuations in the system under investigation. Intensity correlations and sub-Poisson statistics were observed with two lasers in EIT regime 4, 10]. In nonlinear magneto-rotation [11], quadrature squeezing theoretically predicted [12] has been experimentally demonstrated [14].

In the present experiment, we have two beams of strong laser radiation with different polarizations interacting with the atomic system (as shown in Fig. 1a). There are two mechanisms by which the intensity fluctuations can be generated in an atomic medium. One is related to the phase fluctuations of laser field (a linear effect) [15] and the second is four-wave mixing of vacuum modes (a non-linear effect) [17]. It was shown that the efficiency of phase noise (PhN) conversion to intensity noise (IN) is smaller at higher intensity, when non-linear processes are dominant. The PhN-to-IN conversion phenomenon was used for noise spectroscopy of resonance medium [15]. Noise spectroscopy of EIT is studied in 16. Also excess noise was observed under EIT conditions. We studied correlations on delay time and dependence of the maximal correlation on two-photon detuning. In our case optical density has been large and noise spectrum is defined by the shape of spectral hole due to one-photon saturation [9].

Physical insight into this process is gained on the basis of a simplified approach treating the laser fields clasically, using the density matrix for treating the resonance atomic responce, and taking into account propagation that are very essential for the problem. The laser beams are in the resonance three-level medium as depicted in Fig. 1.

Indeed, the polarizations and coherence in the three-level system are given [18] by

$$
\begin{array}{r}
\rho_{c b}=-\frac{\Gamma_{c a}+\Gamma_{a b}}{2 \Gamma_{c a} \Gamma_{a b}} \frac{\Omega_{1} \Omega_{2}}{\Gamma_{c b}+\frac{\left|\Omega_{2}\right|^{2}}{\Gamma_{a b}}+\frac{\left|\Omega_{1}\right|^{2}}{\Gamma_{c a}}}, \\
\rho_{a b}=-i \frac{n_{b a} \Omega_{1}+\rho_{c b} \Omega_{2}}{\Gamma_{a b}}, \quad \rho_{c a}=i \frac{n_{c a} \Omega_{2}+\rho_{c b} \Omega_{1}}{\Gamma_{c a}}
\end{array}
$$

where $\Gamma_{a b}=\gamma_{a b}+i\left(\omega_{a b}-\nu_{1}\right) ; \Gamma_{c a}=\gamma_{c a}-i\left(\omega_{a c}-\nu_{2}\right) ; \Gamma_{c b}=$ $\gamma_{c b}+i\left(\omega_{c b}-\nu_{1}+\nu_{2}\right)$; where $\omega_{\alpha \beta}$ are the atomic frequencies, and $\nu_{1}(t)=\nu_{2}(t)$ are the instant frequency of laser radiation in both beams having orthogonal polarizations. Practically all population is destributed between levels $b$ and $c, n_{b}=$ $n_{c} \simeq 1 / 2$, no population is in level $a, n_{a}=0$. The equations for field propagation are

$$
\frac{\partial \Omega_{1}}{\partial z}=-i \eta_{b} \rho_{a b}, \quad \frac{\partial \Omega_{2}}{\partial z}=-i \eta_{c} \rho_{a c}
$$

where $\eta_{b}=\nu_{1} N \wp_{b} /\left(2 \epsilon_{0} c\right), \eta_{c}=\nu_{2} N \wp_{c} /\left(2 \epsilon_{0} c\right)$ are the coupling constants, $N$ is the density of medium, $\epsilon_{0}$ is the permitivity of the vacuum, and $c$ is the speed of light in vacuum.

Depending on the one- and two-photon detunings the amplitude fluctuations for fields occurs in phase or $\pi$ out of phase. When two fields are in the resonance with atomic transitions, phase fluctuation of laser field leads to detuning from the resonance, but one-photon detunings for both fields are the same, so the change of intensities are in the same direction, and the fields are correlated. When the detuning is included, then the change of absorption is different for these two beams. One field frequency is tuning closer to the resonance but another is tuning out of resonance. The last gives rise for anti-correlations. The time scale to establish correlation or anti-correlation between intensities of laser beams depends on long-lived coherence relaxation between magnetic sublevels. An intersting result is that the spectral width of correlation function is more tolerant to the power broadening giving us 4 times norrower peak than the EIT width. The second mechanism, using four-wave mixing, gives us a nonlinear contribution to the intensity fluctuations. Let us note that both mechanisms leads to correlation at the EIT condition; correlated intensities can be also considered from the point of view of matched pulses [19].

A useful theoretical approach for this situation is the technique developed for the CEL 7, 8]. The system is practically the same, but instead of $\mathrm{V}$-scheme we have here Lambdasystem, and the basic processes, described in term of the CEL analysis, is locking and unlocking. The basic equation describing the obtain results for the angle $\theta$, which is the phase deference of two radiation modes, is given by

$$
\dot{\theta}=a-b \sin \theta+\mathcal{F}(\theta)
$$


where $\mathcal{F}(\theta)=\cos \frac{\theta}{2} F_{-}(\theta)+\sin \frac{\theta}{2} F_{+}(\theta), F_{ \pm}(\theta)$ are the Langevin forces. When two modes are locked their intensities start correlating [8].

We performed numerical simulations by using the quantum regression theory taking the same values of the parameters as in the current experiments and the results will be published elsewhere 20].

The the correlation peak in Fig. 1b is quite narrow, just $\tau=18$ ns. The corresponding frequency spectrum has a width $18 \mathrm{MHz}$ that is an order more then EIT width 1.2 $\mathrm{MHz}$. We have observed the noise spectrum of laser radiaiton after the cell directly with a spectrum analyzer. Probably correlated spectral components of coupled optical fields find appropriate degenerated $\Lambda$ systems. The magnetic field changes correlations between the waveforms. In Fig. 1c one can see the anti-correlation peak and some fluctuations near zero level ( $B=-0.47$ Gauss). On the slope of the EIT resonance phase noise should be more efficiently converted to the intensity noise. In Figure 2 one can see that the noise on the slope of the EIT resonance, more pronounced to compare with at the EIT resonance.

In conclusion, we report an experimental observation of intensity correlations and anti-correlations of coupled fields in a dense $\mathrm{Rb}$ vapor where lower level coherence is created between Zeeman sub-levels by two laser beams with orthoganal polarizations. Intensity fluctuations induced by resonant medium are correlated under resonance EIT condition and anti-correlated at some value of two photon detuning. Narrow correlation and anti-correlations peaks are associated with frequencies above EIT width and natural optical width. Dependence of correlations on magnetic field (two -photon detuning) show resonance behavior. The resonances are near 4 times narrower than the width of the observed EIT resonances.

Correlation properties of coupled fields in $\Lambda$ scheme can be used to reduce noise and improve performance of EIT based atomic clocks and magnetometers. The PhN-to-IN conversion is an important physical process limiting the accuracy. In EIT atomic clock and magnetometers [21], it is possible to avoid the contribution of the atomic medium induced excess intensity noise. In $\Lambda$-EIT we demonstrated that waveforms of transmitted optical field can be strongly correlated. If one would detect coupled fields separately by two independent photo-detectors and then subtract signals, the noise would be reduced to photonic shot noise or even less (sub-Poissonian photon statistics of coupled optical fields).

We thank G. Ariunbold, S.E. Harris, A. Patnaik, Z.E. Sariyanni, A.S.Zibrov for useful and fruitful discussions, V.V.Vasiliev for his help with external cavity laser, H.Chen for his help with Lab View and gratefully acknowledge the support from the Office of Naval Research, the Air Force Re- search Laboratory (Rome, NY), Defense Advanced Research Projects Agency-QuIST, Texas A\&M University Telecommunication and Information Task Force (TITF) Initiative, and the Robert A. Welch Foundation (Grant No. A-1261).

[1] C.H. van der Wal, M.D. Eisaman, A. Andre, R.L. Walsworth, D.F. Phyllips, A.S. Zibrov, M.D. Lukin, Science 301, 196 (2003).

[2] A. Kuzmich, W.P. Bowen, A.D. Boozer, A. Boca, C.W. Chou, L.M. Duan, H.J. Kimble, Nature 423, 731 (2003).

[3] V. Balic, D.A. Braje, P. Kolchin, G.Y. Yin, S.E. Harris, Phys. Rev. Lett. (2005)

[4] C. G. Alzar, L. Cruz, J. A. Gomez, M. F. Santos, and P. Nussenzveig, Europhys. Lett. 61, 485 (2003).

[5] H. Xiong, M.O. Scully, M.S. Zubairy, Phys. Rev. Lett. 94, 023902 (2005).

[6] M. Hennrich, A. Kuhn, and G. Rempe, Phys. Rev. Lett. 94, 053604 (2005).

[7] M.O. Scully, Phys. Rev. Lett. 55, 2802 (1985); M.O. Scully, M.S. Zubairy, Phys. Rev. A35, 752 (1987).

[8] W. Schleich, M. O. Scully, and H.-G. von Garssen, Phys. Rev. A 37, 3010 (1988); W. Schleich and M. O. Scully, Phys. Rev. A $37,1261(1988)$

[9] A.M. Akulshin, V.A. Sautenkov, V.L. Velichansky, et al., Opt. Commun. 77, 295 (1990).

[10] B.L. Lu, W.H. Burkett, M. Xiao, Phys. Rev. A56, 976 (1997); A.F. Huss, R. Lammegger, C. Neureiter, E.A.Korsunsky, and L.Windholz, Phys. Rev. Lett., 93, 223601 (2004); E.E. Mikhailov, V.A. Sautenkov, Yu.V. Rostovtsev, A. Zhang, M. S. Zubairy, M. O. Scully, G. R. Welch http://arxiv.org/abs/quant-ph/0503085

[11] D. Budker, W. Gawlik, D.F. Kimball, S.M. Rochester, V.V. Yashchuk, A. Weis, Rev. Mod. Phys. 74, 1153 (2002).

[12] A.B. Matsko, I. Novikova, G.R. Welch, D. Budker, D.F. Kimball, S.M. Rochester, Phys. Rev. A 66, 043815 (2002);

[13] D. Akamatsu, K. Akiba, M. Kozuma, Phys. Rev. Lett. 92, 203602 (2004).

[14] Ries J, Brezger B, Lvovsky AI, Phys. Rev. A 68, 025801 (2003)

[15] T. Yabuzaki, T. Mitsui, U. Tanaka, Phys. Rev. Lett. 67, 2453 (1991).

[16] M. Martinelli, P. Valente, H.Failache, et al. Phys. Rev. A69, 043809 (2004)

[17] W. V. Davis, M. Kauranen, E. M. Nagasako, R. J. Gehr, A. L. Gaeta, and R. W. Boyd, Phys.Rev. A 51 (1995)

[18] M. O. Scully and M. S. Zubairy, Quantum Optics, Cambridge University Press, Cambridge, England, (1997).

[19] S. E. Harris, Phys. Rev. Lett. 70, 552-555 (1993).

[20] Our work is close to that of A. Patnaik, M.O. Scully, in preparation.

[21] M. Fleishhauer, M.O. Scully, Phys. Rev. A49, 1973 (1994); J. Kitching, S. Knappe, L. Hollberg, Appl. Phys. Lett. 81, 553 (2002); P.D.D. Schwind, S. Knappe, V. Shah, et al., Appl. Phys. Letts., 85, 6409, (2004). 